\title{
Information Encryption Based on Using Arbitrary Two-Step Phase-Shift Interferometry
}

\author{
Chi-Ching Chang ${ }^{1}$, Wen-Ho Wu ${ }^{2}$, Min-Tzung Shiu ${ }^{2}$, Wang-Ta Hsieh ${ }^{2}$, Je-Chung Wang ${ }^{3}$, Hon-Fai Yau ${ }^{4}$ \\ ${ }^{1}$ Institute of Electro-Optical \& Energy Engineering, Ming Dao University, Chinese Taipei \\ ${ }^{2}$ School of Defense Science, National Defense University, Chinese Taipei \\ ${ }^{3}$ Department of Applied Chemistry and Materials Science, National Defense University, Chinese Taipei \\ ${ }^{4}$ Department of Optics and Photonics, National Central University, Chinese Taipei \\ E-mail: chichang@mdu.edu.tw \\ Received August 4, 2011; revised November 8, 2011; accepted November 16, 2011
}

\begin{abstract}
A deterministic phase-encoded encryption system is proposed. A lenticular lens array (LLA) sheet with a particular LPI (lenticular per inch) number is chosen as a modulator (key) instead of the random phase modulator. The suggested encryption scheme is based on arbitrary two-step phase-shift interferometry (PSI), using an unknown phase step. The encryption and decryption principle is based on an LLA in arbitrary unknown two-step PSI. Right key holograms can be used to theoretically show that the object wavefront is the only one left in the hologram plane and that all accompanying undesired terms are eliminated. The encrypted image can therefore be numerically and successfully decrypted with the right key in the image plane. The number of degrees of freedom of the encryption scheme increases with the distance from the object and the LLA to the CCD, and also with the unknown phase-step and the LLA LPI number. Computer simulations are performed to verify the encryption and decryption principles without a key, with the wrong key and with the right key. Optical experiments are also performed to validate them.
\end{abstract}

Keywords: Deterministic Phase-Encoded Encryption, Arbitrary Two-Step Phase-Shift Interferometry, Lenticular Lens Array

\section{Introduction}

In digital holography (DH), the hologram is digitized and the object wavefront is numerically reconstructed using a computer. This idea was proposed by Goodman and Lawrence [1]. This approach has attracted increasing attention because a charged-couple device (CCD) camera was first used to acquire holograms directly with off-axis and in-line configurations $[2,3]$. This method, $\mathrm{DH}$, enables full digital hologram recording and processing without chemical or physical development, increasing the flexibility and speed of the experimental process.

Since the mid-1990s, DH has found several practical and successful applications, including deformation analysis, particle tracking, microscopy, and encrypting information. One such DH application is the encryption of information, which is done using various algorithms and architectures [4-25], such that an unauthorized person cannot easily access the secret information. The basic principle of this encryption method is to encode the ob- ject wavefront, the reference wavefront or a combination of both wavefronts by positioning phase encoding components in the input, Fresnel, or a Fraunhofer domain, or a combination of both domains. Of the encryption approaches that have been developed in recent years, the most often used and highly successful ones are based on phase-shift interferometry (PSI), making good use of available CCD resources to capture on-axis encrypted digital holograms $[6-8,12,14]$.

Since the standard PSI approach depends on a special and constant phase shift, $2 \pi / N$, where the integer $N \geq 3$, this requirement is commonly difficult to meet precisely in practice. Therefore, Meng et al. $[17,20]$ presented an algorithm for two-step PSI, with an arbitrary known phase step $\delta$, for image encryption with double random-phase encoding in the Fresnel domain.

Unlike other works $[17,20]$, this work proposes a deterministic phase-encoded encryption system that uses an LLA as a phase modulator (key) [22]. The encryption scheme is based on the arbitrary two-step PSI [26], but 
with an arbitrary unknown phase step $\Delta \varphi$. The LLA is placed in the reference path to modulate and thus encode the incident plane wave phase, which is reflected by a mirror. The phase-encoded wavefront is then used as the reference wave in encrypting the object wavefront.

The unknown phase-step $\Delta \varphi$ of a reference wave is controlled by inserting a glass plate between the mirror and the LLA. The unknown $\Delta \varphi$ can be estimated from Ref. 26, with the assumption that the phase change due to object illumination by the object beam is negligible. This assumption obviously applies to image encryption. Notably, the amplitude of the reference wave can be greater or less than that of the object wave in the unknown two-step PSI.

The encrypted hologram includes the de term, virtual image and twin image, because the object wavefront interferes with the phase-encoded reference wave at the hologram plane. The dc term and twin image (unwanted terms) can usually be eliminated by applying the principle proposed in the cited work [26]. However, the virtual image is then the product of the object wavefront and the conjugate term of the phase-encoded reference wave. If the conjugate term of the phase-encoded reference wave is not accurately decoded from the virtual image. Then the encrypted image cannot be decrypted and faithfully recovered. The conjugate of the phase-encoded term can only be completely cancelled and counteracted by the phase-encoded term, which can be retrieved from two right key holograms (with no object) using the algorithm proposed in the work cited above [26]. Two right key holograms are therefore required to implement the decryption scheme and the encrypted image is numerically decrypted and recovered satisfactorily.

The rest of this paper is organized as follows. Section 2 briefly reviews the physical properties of the phaseencoded LLA component. Section 3 then elucidates the encryption and decryption principle with an LLA in arbitrary unknown two-step PSI. In Section 4 computer simulations are performed to confirm the encryption and decryption scheme without a key, with the wrong key and with the right key. In Section 5 Mach-Zehnder interferometry is used to implement the encryption system and to validate the scheme. For comparison, numeric decryptions of the encrypted information carried by the object wavefront without a key, with the wrong key and with the right key, are demonstrated. Conclusions are finally drawn in Section 6, along with recommendations for future research.

\section{Brief Review of Phase-Encoded Component-LLA}

An LLA sheet is used to regulate the reference beam phase to perform the encryption scheme phase-encoded modulations. The LLA is comprised of several cylindrical lenses of equal size and curvature, formed into a one-dimensional array [27] (Figure 1).

Notably, the phase transformation of LLA can be expressed as [28]

$$
t_{L L A}(\xi, \eta ; m, l)=\exp \left[j k(n-1) \frac{(\xi-m l)^{2}}{2 \mathrm{R}}\right]
$$

where $m=0, \pm 1, \pm 2, \pm 3, \cdots$, which can be calculated by taking the integral of the ratio $x / l, m=\operatorname{int}(x / l)$. The parameters $k, n$ and $R$ are the light source wave numbers $(k$ $=2 \pi / \lambda)$, the refraction index and the lens radius of curvature, respectively. The value $l$ is given by $l=1 / \mathrm{LPI}$, where LPI is the LLA density.

The correlation $s(x, y)$ between two phase functions $t_{L L A}(\xi, \eta ; m, l)$ and $t_{L L A}(\xi, \eta ; m, l$ ') [29],.

$$
s(\xi, \eta)=t_{L L A}(\xi, \eta ; m, l) \otimes t_{L L A}\left(\xi, \eta ; m^{\prime}, l^{\prime}\right)
$$

where the operator $\otimes$ denotes correlation, and can be evaluated. Various phase-encoded schemes can be easily shown implemented using different LPI numbers, such as LPI $=50,62,75$ and others [30]. Additionally, the phase code value and length can be varied and realized using various LPI numbers. Figures 2(a) and 2(b) present the autocorrelations of 62 LPI and 50 LPI LLA phase functions. Figure 2(c) presents the cross-correlation between the two functions. The phase code length equals 1392 . Under the ideality assumption [27] the parameters of the two LPI LLAs used to evaluate the correlations $s(\xi, \eta)$ are presented in Table 1 [30].

Table 1. Parameters of 50 and 62 LPI LLA.

\begin{tabular}{ccc}
\hline & 50 LPI & 62 LPI \\
\hline $\mathrm{n}$ & 1.58 & 1.58 \\
width of lenticule $(\mathrm{cm})$ & 0.0508 & 0.0408 \\
$\mathrm{R}(\mathrm{cm})$ & 0.0254 & 0.0204 \\
\hline
\end{tabular}

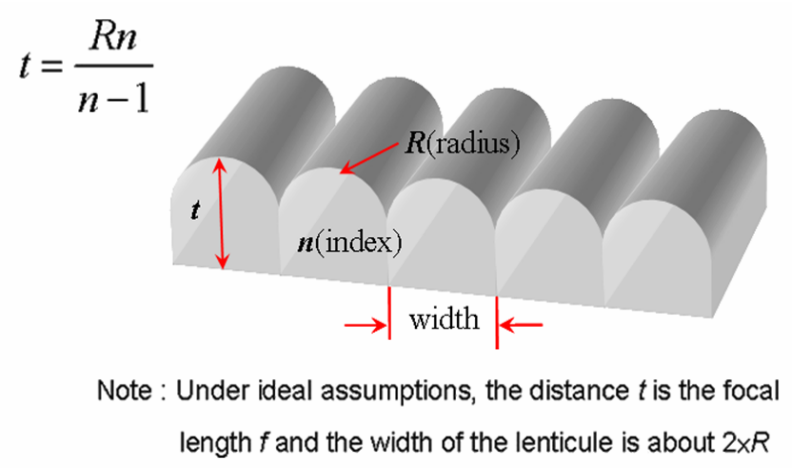

Figure 1. Side view of a lenticular lens array. 


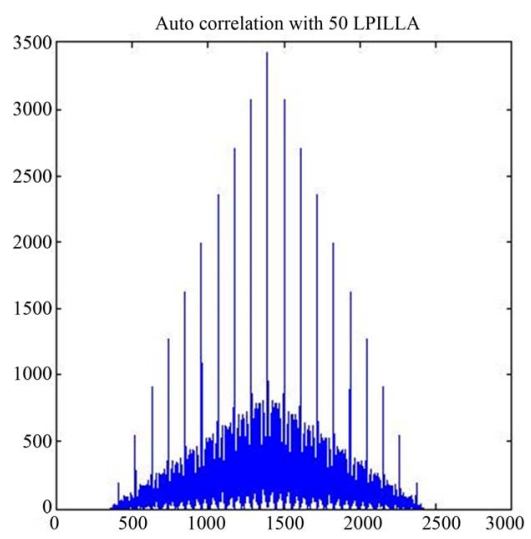

(a)

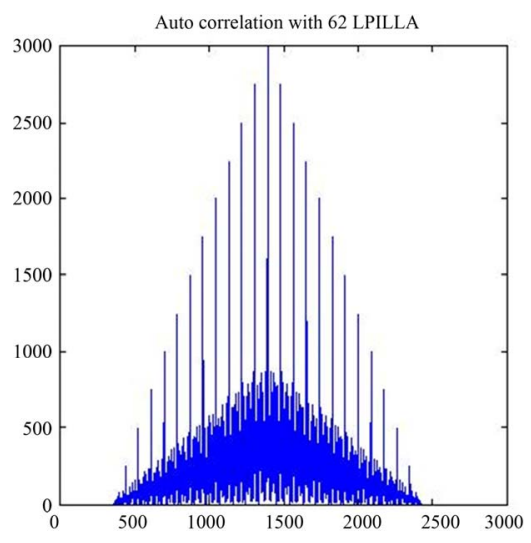

(b)

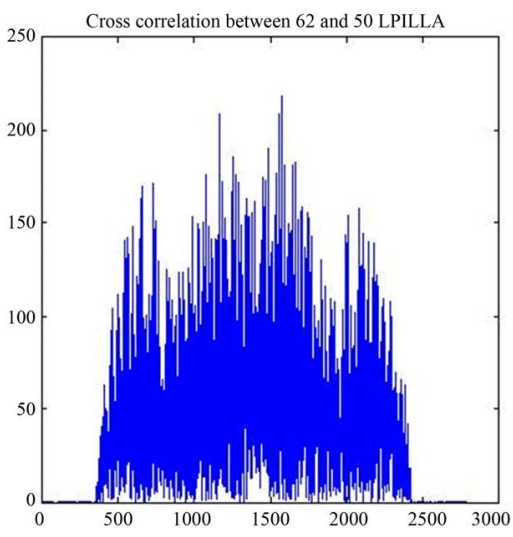

(c)

Figure 2. Evaluations of correlation: (a) autocorrelation with 50 LPI LLA phase function; (b) autocorrelation with 62 LPI LLA phase function; (c) cross-correlation between two phase functions.

Based on Figures 2(a)-(c) the encrypted information, which is encoded using an LLA with a particular LPI number, can only be decrypted using that (correct) LPI number, which is the right key. It cannot be recovered without a key or with the incorrect LPI number. For example, if the encrypted image is encoded using a 62 LPI LLA, decoding encrypted information without a key or with a 50 LPI LLA is impossible. The number of degrees of freedom of the encryption scheme increases with the LLA LPI number.

\section{Principle of Encryption and Decryption using Arbitrary Unknown Two-Step PSI}

In the proposed encryption scheme (Figure 3) a reference wave with known amplitude and phase, is modulated using an LLA with a particular LPI. The modulated reference wave is used as the deterministic phase- encoded reference wave. The object wavefront is generated using a light that passes through or reflects from the object.

Let $\psi_{O}$ and $\psi_{D P M}(x, y ; m, l)$ denote the object wavefront to be recorded and the phase-encoded reference wave at the hologram plane, respectively. The $\psi_{D P M}(x, y$; $m, l)$ can be mathematically expressed from the LLA modulation and distance $z$ propagation as [31]

$$
\begin{aligned}
& \psi_{D P M}(x, y ; m, l)= \\
& \frac{\exp (j k z)}{j \lambda z} \iint t_{L L A} \exp \left\{\frac{j k}{2 z}\left[(\xi-x)^{2}+(\eta-y)^{2}\right]\right\} \mathrm{d} \xi d \eta
\end{aligned}
$$

where $(x, y)$ denote coordinates in the hologram plane.

The intensity of the first encrypted hologram at the hologram plane is

$$
\begin{aligned}
I_{E N C I}(x, y ; m, l)=\left|\psi_{O} \psi_{D P M}\right|^{2}= & \left|\psi_{O}\right|^{2}+\left|\psi_{D P M}\right|^{2} \\
& +\psi_{O} \psi_{D P M}^{*}+\psi_{O}^{*} \psi_{D P M}
\end{aligned}
$$

On the right-hand side of Equation (4) the first two

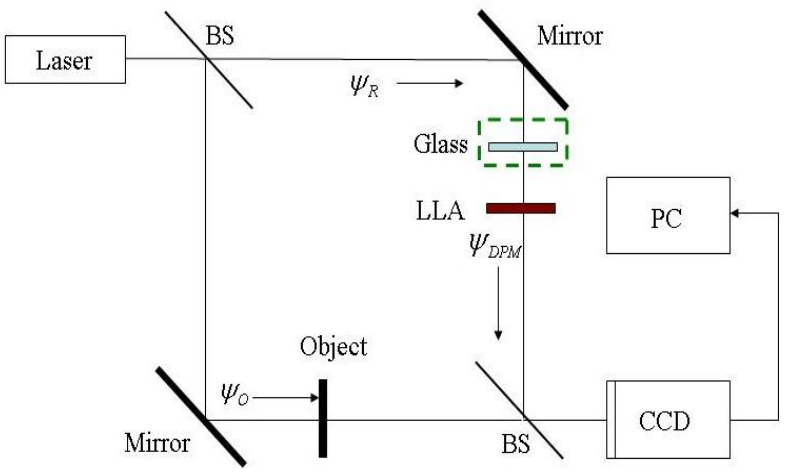

Figure 3. Schematic of the proposed encryption setup.

terms constitute the de term, the third term corresponds to the virtual image and the fourth corresponds to the real image. Only the third term contains the desired information to be decrypted. The other terms, which cause blurring, must be removed and (or) suppressed before numerical reconstruction. The unwanted terms are suppressed based on the principle in the work cited above [26].

The dc term can be completely eliminated in typical method [32], which separately captures the intensities of the object wave and reference wave at the hologram plane. The reduced intensity of the first hologram can be written as

$$
\begin{aligned}
I_{E N C 2}(x, y ; m, l)= & \left|\psi_{O}+\psi_{D P M} \exp (j \Delta \phi)\right|^{2} \\
= & \left|\psi_{O}\right|^{2}+\left|\psi_{D P M}\right|^{2} \\
& +\psi_{O} \psi_{D P M}^{*} \exp (-j \Delta \phi) \\
& +\psi_{O}^{*} \psi_{D P M} \exp (+\Delta \phi)
\end{aligned}
$$

An unknown phase-step $\Delta \varphi$ of the reference wave is obtained by inserting a glass plate in the reference path. The intensity of the second encrypted hologram can then be expressed as 


$$
\begin{aligned}
I_{E N C 2}(x, y ; m, l)= & \left|\psi_{O}+\psi_{D P M} \exp (j \Delta \phi)\right|^{2} \\
= & \left|\psi_{O}\right|^{2}+\left|\psi_{D P M}\right|^{2} \\
& +\psi_{O} \psi_{D P M}^{*} \exp (-j \Delta \phi) \\
& +\psi_{O}^{*} \psi_{D P M} \exp (+\Delta \phi)
\end{aligned}
$$

Similarly, when the dc term is completely suppressed, the reduced intensity of the second hologram is given by

$$
\begin{aligned}
I_{E N C 2}^{\prime}(x, y ; m, l)= & \psi_{O} \psi_{D P M}^{*} \exp (-j \Delta \varphi) \\
& +\psi_{O}^{*} \psi_{D P M} \exp (+\Delta \varphi)
\end{aligned}
$$

From the simple argument in the cited work [26], the unknown phase-step can be easily estimated with limited pixels near the optical axis (in discrete form) as

$$
\frac{I_{E N C 2}^{\prime}(p, q)}{I_{E N C l}^{\prime}(p, q)} \cong \cos [\Delta \varphi(p, q)]
$$

where $p=1, \ldots, M$ and $q=1, \ldots, N$ and $M \times N$ is the number of light-sensitive pixels on the CCD, respectively. Alternatively, $\Delta \varphi$ can be estimated using the Fourier transform ratio of their reduced intensities with the same approximations,

$$
\frac{\mathfrak{J}\left\{I_{E N C 2}^{\prime}\right\}}{\mathfrak{I}\left\{I_{E N C 1}^{\prime}\right\}} \cong \cos (\Delta \varphi)
$$

Let Equation (7) be multiplied by the term $\exp (-j \Delta \varphi)$ and then subtracted from Equation (5). The following equation is obtained.

$$
\begin{aligned}
I_{E N C l}^{\prime}-\exp (-j \Delta \varphi) I_{E N C 2}^{\prime} & =[1-\exp (-j 2 \Delta \varphi)] \\
& \psi_{O} \psi_{D P M}^{*} \\
& \propto \psi_{O}(p, q) \psi_{D P M}^{*}(p, q ; m, l)
\end{aligned}
$$

since $\Delta \varphi$ can be regarded as fixed in the recording stage in the arbitrary two phase-step approach, the twin-image is satisfactorily and completely eliminated by the numerical operations performed using the reduced intensities of the two holograms and the estimated value of $\Delta \varphi$. However, the right-hand side of Equation (10) is now seen to be composed of the object wavefront $\psi_{O}$ and the conjugate term of $\psi_{D P M}(p, q ; m, l)$, which is abbreviated as $\psi_{D P M}(m, l)$ hereafter. If the conjugate term of $\psi_{D P M}(m$, $l$ ) cannot be determined by decoding the virtual image and thus separated from $\psi_{O}$, the encrypted image will then not to be decrypted or faithfully recovered from the encrypted holograms. To accurately decode the conjugate term of $\psi_{D P M}(m, l)$ from Equation (10), two additional right key holograms must be recorded using the right key, which can be easily and mathematically confirmed by the arguments below. For comparison, the encrypted information about the object wavefront will be numerically decrypted without a key, with a wrong key and with the right key.

\subsection{Decryption without Key}

For decryption with no key, the abbreviation

$$
\left.U_{E N C}(p, q ; m, l)\right|_{h}=\psi_{O}(p, q) \psi_{D P M}^{*}(m, l)
$$

is applied in the hologram plane. Then, let $U_{E N C}$ be directly reconstructed in the image plane using the convolution approach [33]

$$
\left.\psi_{O}(p, q) \psi_{D P M}^{*}(m, l)\right|_{i}=\mathfrak{I}^{-1}\left\{\mathfrak{I}\left\{U_{E N C}\right\} \mathfrak{I}\{h(z=d)\}\right\}
$$

where $\mathrm{h}(z=d)$ represents the impulse response through a distance $z=d$. As expected, the left-hand side of Equation (12) still contains the product of $\psi_{O}$ and the conjugate term of $\psi_{D P M}(m, l)$, so the encrypted image is not successfully decrypted in this case.

\subsection{Decryption with Wrong Key - Incorrect LPI $\operatorname{LLA}\left(m^{\prime} \neq m\right.$ and $\left.l ' \neq l\right)$}

Let two wrong key (incorrect LPI LLA) holograms (with no object), $I_{K E Y I}\left(m^{\prime}, l^{\prime}\right)$ and $I_{K E Y 2}\left(m^{\prime}, l^{\prime}\right)$, be captured by the arbitrary two-step PSI method. According to similar numerical procedures derived from Equations (10)-(12), the phase-encoded pattern term $\psi_{D P M}\left(m^{\prime}, l^{\prime}\right)$ and reconstructed object wavefront can be straightforward written down

$$
\begin{aligned}
&\left.U_{K E Y}\left(m^{\prime}, l^{\prime}\right)\right|_{h}=I_{K E Y I}^{\prime}\left(m^{\prime}, l^{\prime}\right)-\exp (+j \Delta \varphi) I_{K E Y 2}^{\prime}\left(m^{\prime}, l^{\prime}\right) \\
& \propto \psi_{D P M}\left(m^{\prime}, l^{\prime}\right) \\
&\left.\psi_{O}\left(p, q ; m, l, m^{\prime}, l^{\prime}\right)\right|_{i} \\
&=\mathfrak{I}^{-1}\left\{\mathfrak{I}\left\{\psi_{O}(p, q) \psi_{D P M}^{*}(m, l) \psi_{D P M}\left(m^{\prime}, l^{\prime}\right)\right\}\right. \\
&\mathfrak{I}\{h(z=d)\}\}
\end{aligned}
$$

Based on the right-hand side of Equation (14a), if the incorrect LPI LLA (wrong key) is used, the conjugate term of $\psi_{D P M}(m, l)$ will not be fully separated from the object wavefront term. Since the conjugate term cannot be completely cancelled by $\psi_{D P M}\left(m^{\prime}, l^{\prime}\right)$, the encrypted image cannot be recovered from the encrypted holograms with the aid of a wrong key.

\subsection{Decryption with Right Key - Correct LPI LLA $\left(m{ }^{\prime}=m\right.$ and $\left.l '=l\right)$}

Let two wrong key (incorrect LPI LLA) holograms (with no object), $I_{K E Y I}\left(m^{\prime}, l^{\prime}\right)$ and $I_{K E Y 2}\left(m^{\prime}, l^{\prime}\right)$, be captured by the arbitrary two-step PSI method. According to similar numerical procedures derived from Equations (10)-(12), the phase-encoded pattern term $\psi_{D P M}\left(m^{\prime}, l l^{\prime}\right)$ and reconstructed object wavefront can be straightforward written down 


$$
\begin{aligned}
& \left.U_{K E Y}(m, l)\right|_{h}=I_{K E Y 1}^{\prime}(m, l)-\exp (+j \Delta \varphi) I_{K E Y 2}^{\prime}(m, l) \\
& \propto \psi_{D P M}(m, l) \\
& \left.\psi_{O}(p, q)\right|_{i}=\mathfrak{J}^{-1}\left\{\mathfrak{J}\left\{\psi_{O}(p, q) \psi_{D P M}^{*}(m, l) \psi_{D P M}(m, l)\right\}\right. \\
& \mathfrak{J}\{h(z=d)\}\}
\end{aligned}
$$

From Equation (14b), the conjugate term of $\psi_{D P M}(m, l)$ is completely cancelled and counteracted by $\psi_{D P M}(m, l)$, and is therefore fully separated from the object wavefront term in the hologram plane. Clearly, if the correct LPI LLA is used, then theoretically only the reconstructed object wavefront term will be left in the image plane and the accompanying undesired term, which is the conjugate term of $\psi_{D P M}(m, l)$, is eliminated in the hologram plane. Therefore, the encrypted image in this suggested encryption system can be numerically and successfully decrypted using arbitrary two-step PSI and right key holograms.

\section{Computer Simulations}

Simulations are performed to verify the proposed approach. In the simulation procedure, the ratio of the intensity of the object wave to that of the reference wave is assumed to be 1:1. Figure 4(a) presents the original input image of two Chinese characters "Chung Cheng" $(1392 \times 1040$ pixels).The 62 LPI and 50 LPI LLA are used as the right key and the wrong key, respectively. The two LLAs are placed at a distance $d=5 \mathrm{~cm}$ from the hologram plane. The distance between the object plane and the hologram plane is $20 \mathrm{~cm}$ and the illumination wavelength $\lambda=0.6328 \mu \mathrm{m}$. The arbitrary phase-step $\Delta \varphi$ is set to 1.30 radians.

Figures 4(b) and (c) present the two simulated encrypted holograms without a phase-step ( $\Delta \varphi=0$ radians)

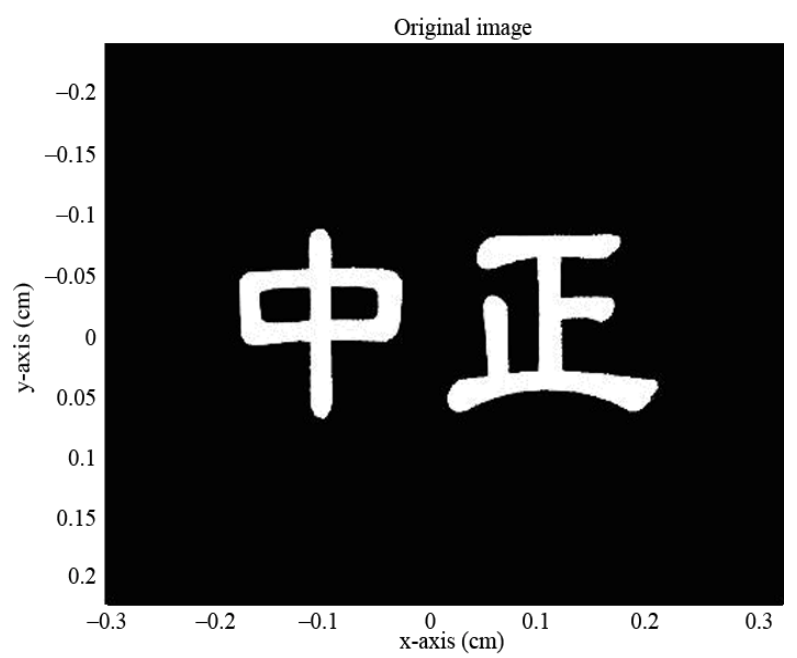

(a) and with an arbitrary phase-step ( $\Delta \varphi=1.30$ radians), respectively. After the dc term is eliminated from the holograms, the $\Delta \varphi$ values can then be estimated using Equation (8) or (9) with the pixel array (515:524, 691:700) close to the optical axis. The optimal value of $\Delta \varphi$ is 1.304 radians (estimated using Equation (9) as presented in Table 2.

Figure 4(d) presents the decrypted magnitude-contrast image, which was directly reconstructed using Equation (12) without a key. As expected, a comparison with the original image does not easily reveal the hidden information, the two Chinese characters, from the decrypted image. Therefore, without further decoding the phase-encoded pattern of the reference wave using the right key, the encrypted information cannot be decrypted.

Figures 4(e) and (f) present the two wrong key holograms (with no object) simulated using the arbitrary two-step ( 0 and 1.30 radians) PSI. The phase-encoded pattern term $\psi_{D P M}\left(m^{\prime}, l^{\prime}\right)$ is obtained numerically using Equation (13a). Since the conjugate term of $\psi_{D P M}(m, l)$ cannot be completely cancelled by $\psi_{D P M}\left(m^{\prime}, l^{\prime}\right)$, it is not fully separated from the object wavefront term in the hologram plane. Figure 4(g) presents the decrypted image numerically reconstructed using Equation (14a) and the wrong key. The hidden information can still not be recovered from the encrypted holograms using the wrong key, as shown in Figure 4(g).

Figures 4(h) and 4(i) present the two right key holograms simulated using the arbitrary two-step ( 0 and 1.30radians) PSI. The phase-encoded pattern term $\psi_{D P M-}$ $(m, l)$ is obtained numerically using Equation (13b). The conjugate term of $\psi_{D P M}(m, l)$ is completely cancelled by $\psi_{D P M}(m, l)$, and now fully separated from the object wavefront term in the hologram plane. Figure 4(j) presents the decrypted magnitude-contrast image that was numerically reconstructed from Equation (14b) using the

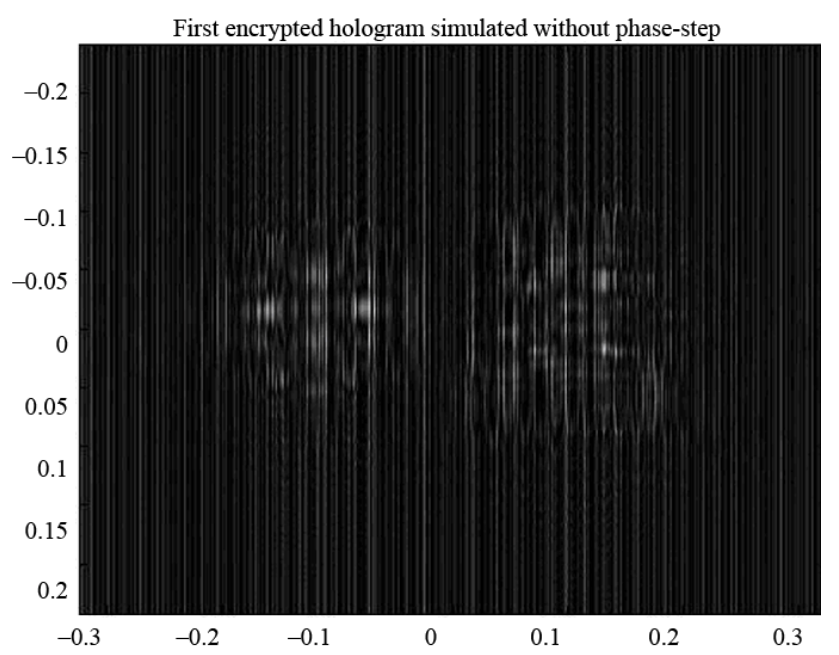

(b) 


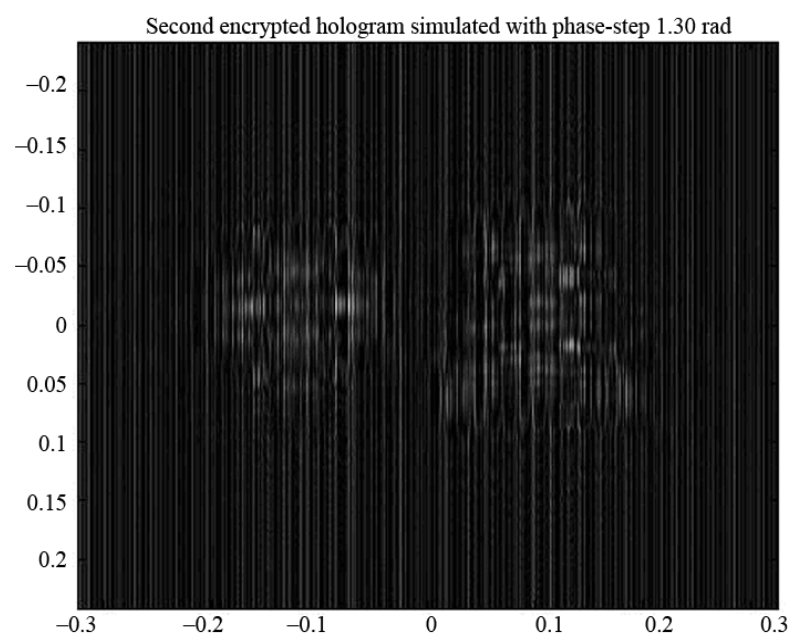

(c)

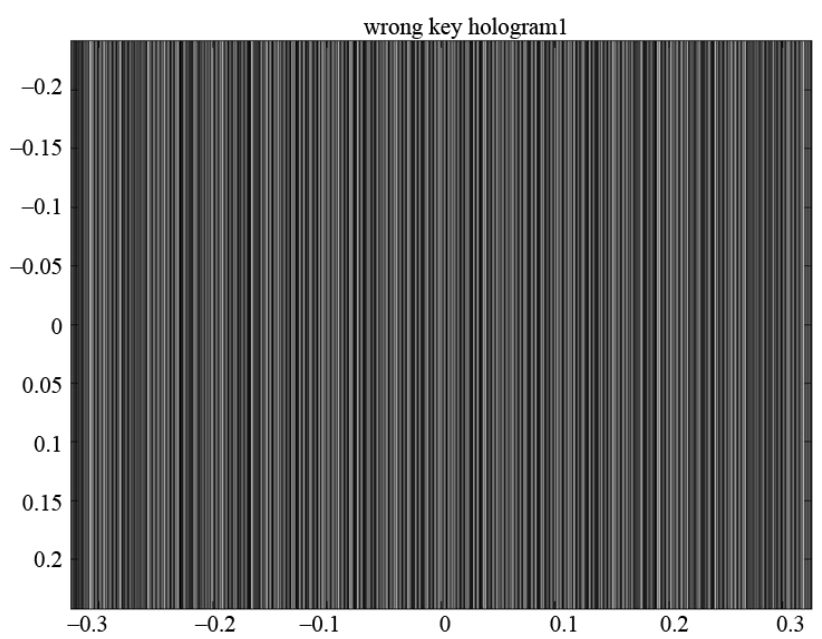

(e)

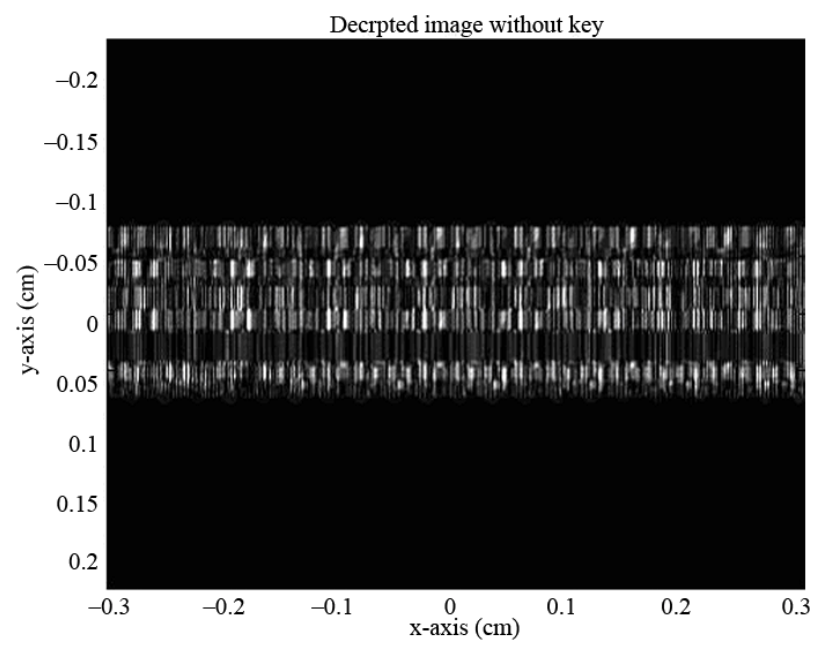

(d)

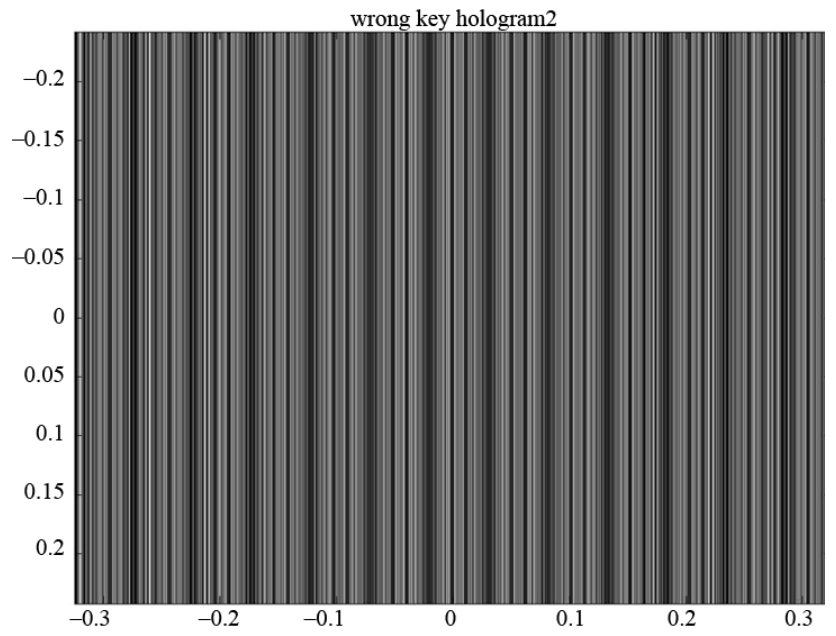

(f)

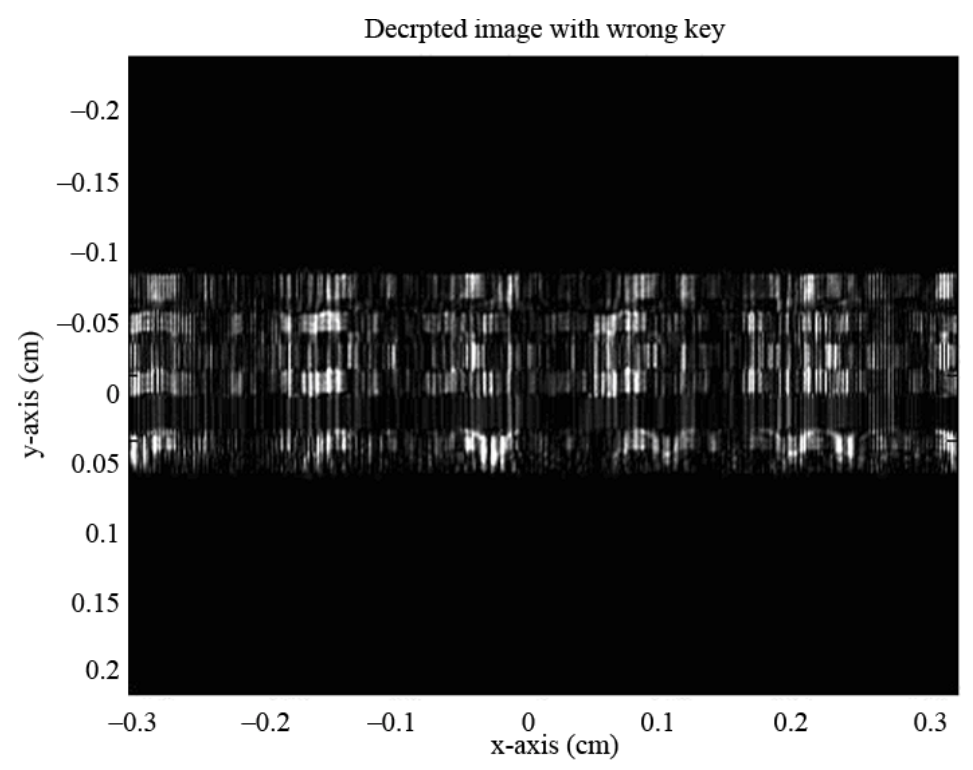

(g) 


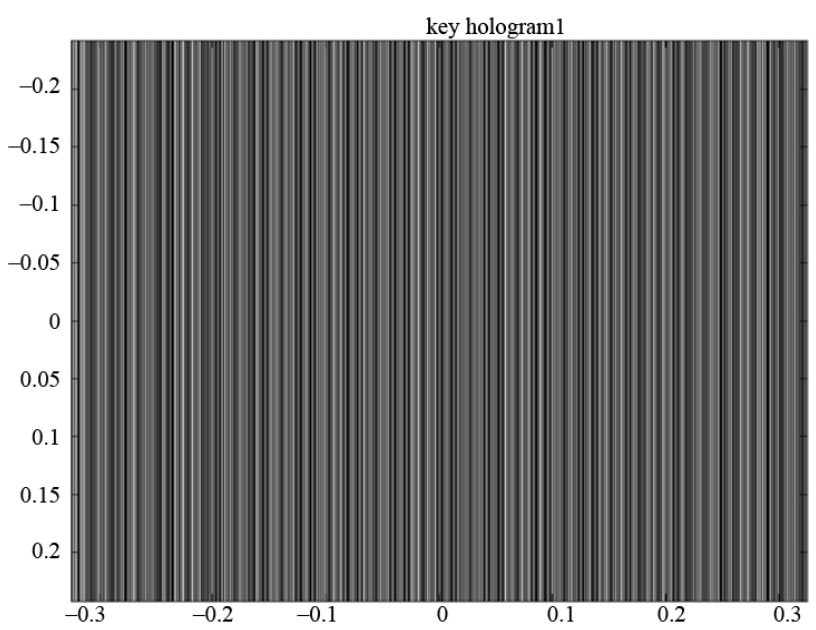

(h)

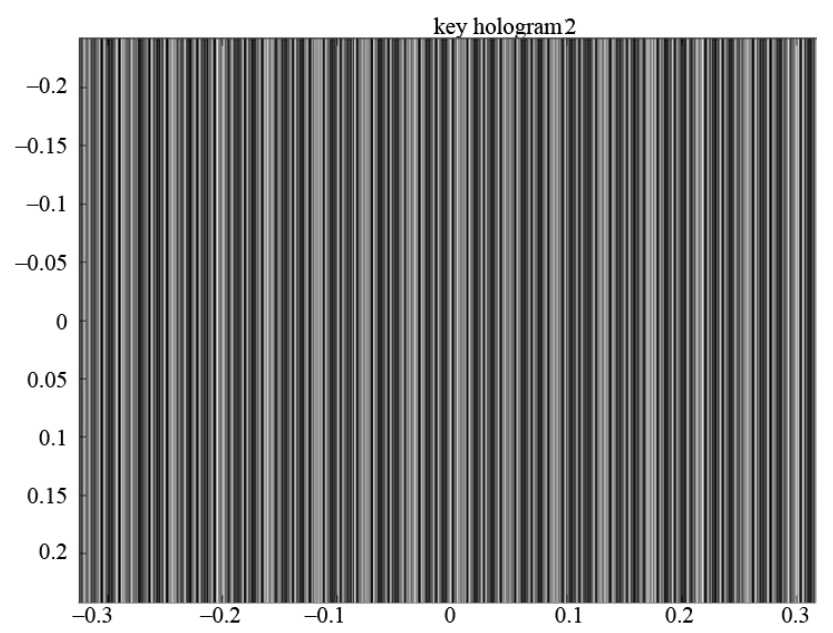

(i)

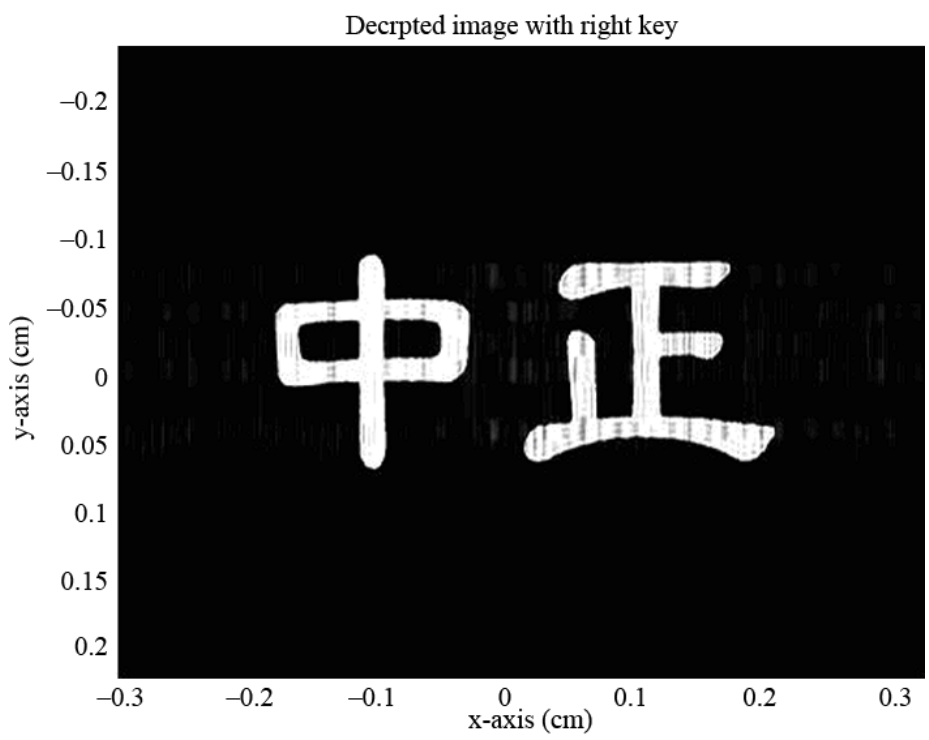

(j)

Figure 4. Simulation results: (a) original input image; (b) first encrypted hologram without phase-step; (c) second encrypted hologram with phase-step 1.30 radians; (d) decrypted image without key; (e) first wrong key hologram without phase-step; (f) second wrong key hologram with phase-step 1.30 radians; (g) decrypted image with wrong key; (h) first right key hologram without phase-step; (i) second right key hologram with phase-step 1.30 radians; (j) decrypted image with right key.

right key. Unlike in Figures 4(d) and (g), the encrypted USAF resolution chart information is clearly identifiable in the center of Figure 4(j), although some artificial blurring is evident. Notably, as determined by comparison with the original image in Figure 4(a), the least square error and SNR (in dB) [34] for Figure 4(j) are around 0.0119 and $19.25 \mathrm{~dB}$, respectively, as indicated in Table 2.

\section{Experimental Setup and Results}

Mach-Zehnder interferometry (Figure 5) was used to realize the encryption system. The light source was a $\mathrm{He}-\mathrm{Ne}$ laser with a power of $20 \mathrm{~mW}$ and a wavelength of
Table 2. Simulations of estimated phase-step value $\Delta \varphi$ and mean square error and SNR of the decrypted images.

\begin{tabular}{cc}
\hline & Arbitrary two-step PSI \\
Default $\Delta \varphi$ (radians) & 1.30 \\
Estimated $\Delta \varphi$ (radians) & 1.304 \\
Error (\%) & $0.3 \%$ \\
Mean square error (magnitude-contrast) & 0.012 \\
SNR (dB) (magnitude-contrast) & 19.25 \\
\hline
\end{tabular}




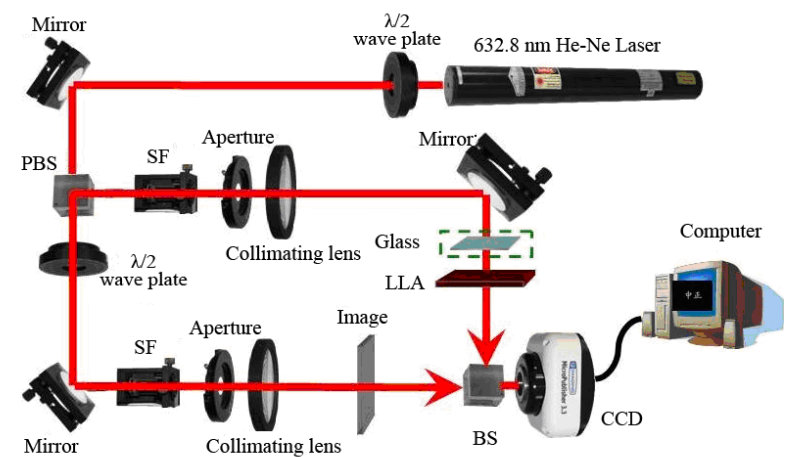

Figure 5. Schematic diagram of the optical encryption setup where SF: spatial filter, PBS: polarized beam splitter, BS: beam splitter.

$\lambda=632.8 \mathrm{~nm}$. Two $\lambda / 2$ retarding wave plates and a polarized beam splitter (PBS) were used together to adjust the object wave intensity ratio to that of the reference wave. Both waves were collimated into a plane wave using spatial filters (SFs), apertures and lenses. Two LLAs, one $62 \mathrm{LPI}$ and the other $50 \mathrm{LPI}$, were used as the right key and wrong key, respectively. Two LLAs were used to modulate the incoming plane wave, which was reflected by a mirror. A light-sensitive sensor (Pixera-150SS CCD camera; $1040 \times 1392$ pixels, $w_{s y}=0.484 \mathrm{~cm}$ and $w_{s x}=0.650$ $\mathrm{cm})$ was used to acquire the holograms.

The phase of the reference wave in the reference path was first left unchanged during the first encrypted hologram recording. An arbitrary phase-step of the reference wave was then applied by inserting a glass plate into the reference path before the second hologram was captured. Two Chinese characters (Figure 6) were encrypted.

The distance between the object and CCD was $d=21.5$ $\mathrm{cm}$. The modulators (LLAs) were placed $7.6 \mathrm{~cm}$ from the CCD. After the object was removed, the wrong key and the right key holograms were obtained by recording the fringe patterns of the modulated reference wave and plane wave using the arbitrary two-step PSI technique.

Figures 7(a) and (b) present the two encrypted holograms without a phase-step and with an arbitrary phasestep, respectively, captured using a CCD in the hologram plane. After the dc term was eliminated from the two encrypted holograms, $\Delta \varphi$ can be estimated using Equation (8) or (9) for the pixel array $(515: 524,691: 700)$ close to the optical axis. The optimal $\Delta \varphi$ value was estimated from Equation (9) to be around 0.1380 radians.

For comparison the encrypted information about the object wavefront was numerically decrypted without a key, with a wrong key and with the right key. Figure 7(c) presents the decrypted magnitude-contrast image that directly reconstructed using Equation (12) without a key. In Figure 7(c) the hidden information is invisible and

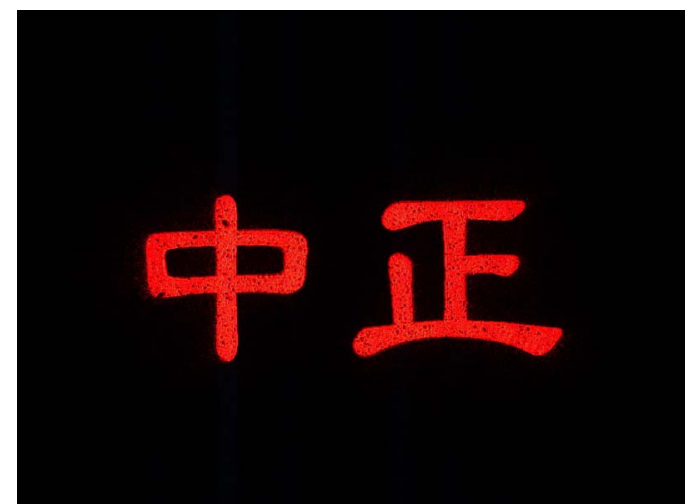

Figure 6. Original optical image to be encrypted.

difficult to identify. Clearly without separating the phase-encoded reference wave pattern from the object wavefront in the hologram plane, the encrypted information in the image plane cannot be decrypted.

Figures 7(d) and (e) present the two wrong key (50 LPI LLA) holograms (with no object) without and with the arbitrary phase-step, respectively. The phase-encoded pattern term $\psi_{D P M}\left(m^{\prime}, l^{\prime}\right)$ is then obtained numerically using Equation (13a) but the effect of the conjugate term of $\psi_{D P M}(m, l)$ cannot be completely cancelled and thereby fully separated from object wavefront term. Figure 7(f) presents the decrypted magnitude-contrast image that was numerically reconstructed using Equation (14a) and a wrong key. Therefore, the hidden information cannot be recovered from the encrypted holograms with the aid of the wrong key.

Figures 7(g) and (h) present the two right key (62 LPI LLA) holograms without and with the arbitrary phasestep, respectively. Similarly, the phase-encoded pattern term $\psi_{D P M}(m, l)$ is obtained using numerical operations from Equatioin (13b). Figure 7(i) presents the decrypted magnitude-contrast image that was numerically reconstructed using Equation (14b) using the right key. Unlike in Figure 7(c) and (f), Figure 7(i) clearly shows the encrypted "Chung Cheng" information. As expected, the conjugate term of $\psi_{D P M}(m, l)$ is cancelled by $\psi_{D P M}(m, l)$ and separated from the object wavefront term in the hologram plane. Clearly, when the right key is used, the undesired term, which is, the conjugate term of $\psi_{D P M}(m$, $l$ ), is eliminated in the hologram plane and only the reconstructed object wavefront term remains in the image plane. The hidden information is numerically decrypted using arbitrary two-step PSI with right key holograms

\section{Conclusions}

This work presented a deterministic phase-encoding encryption system that uses a lenticular lens array (LLA) as a phase modulator. The encryption approach was imple 


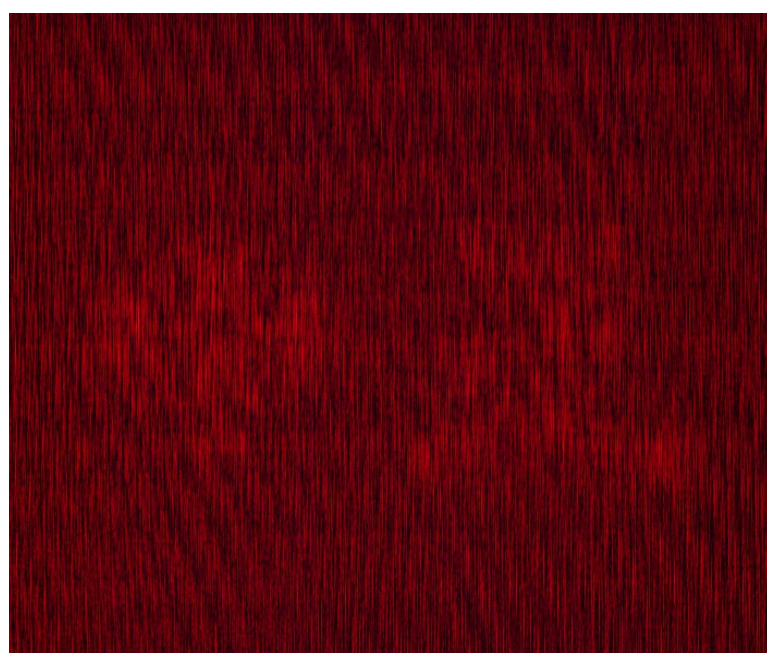

(a)

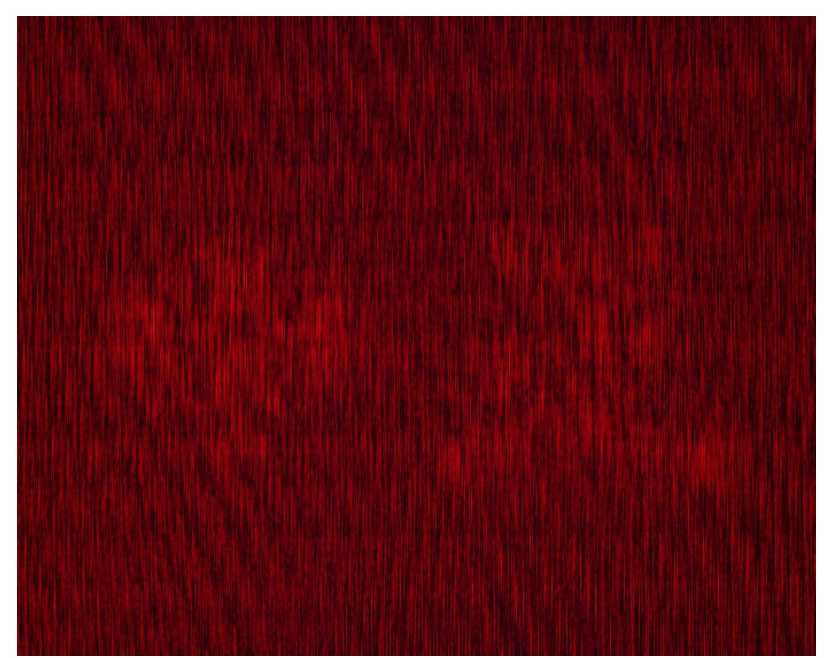

(b)

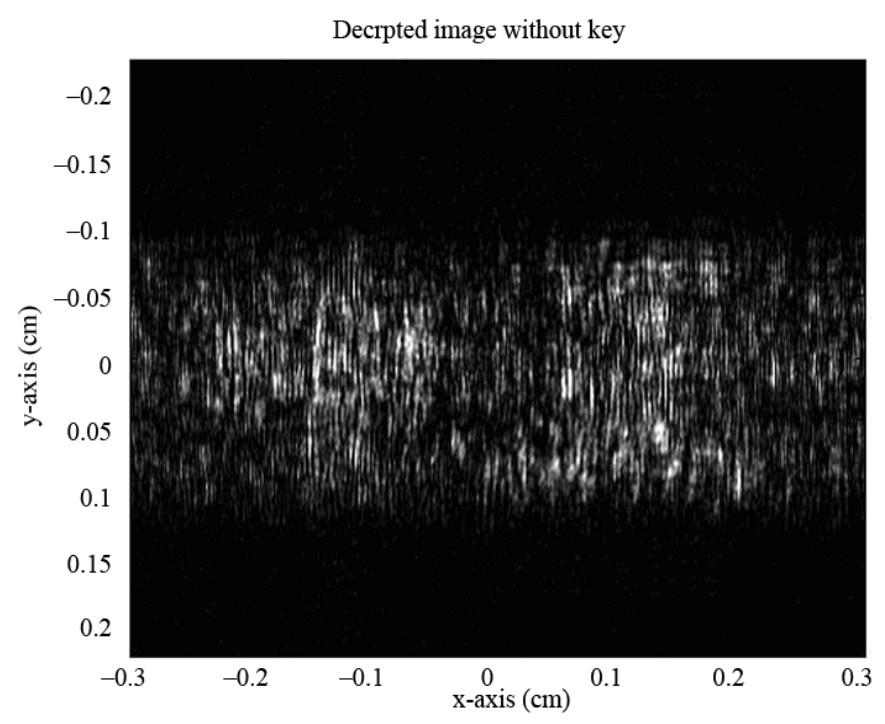

(c)

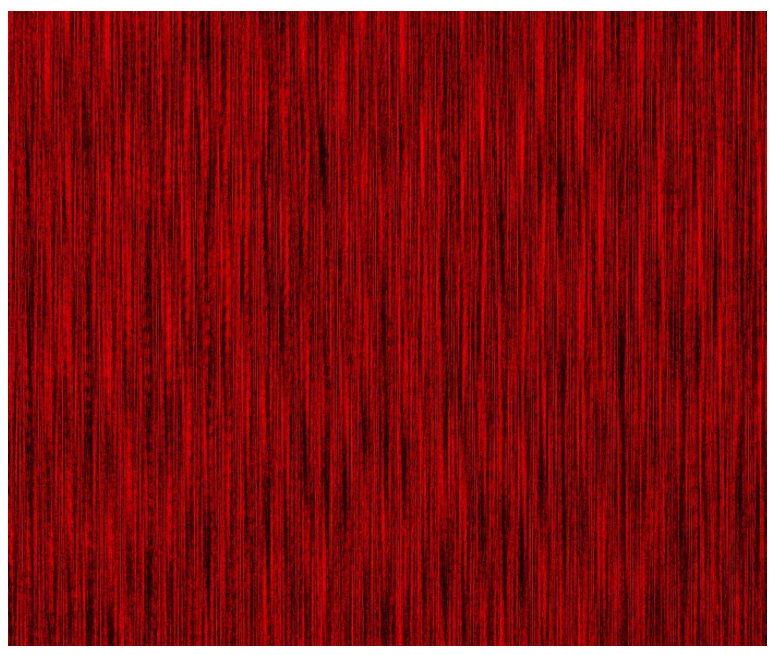

(d)

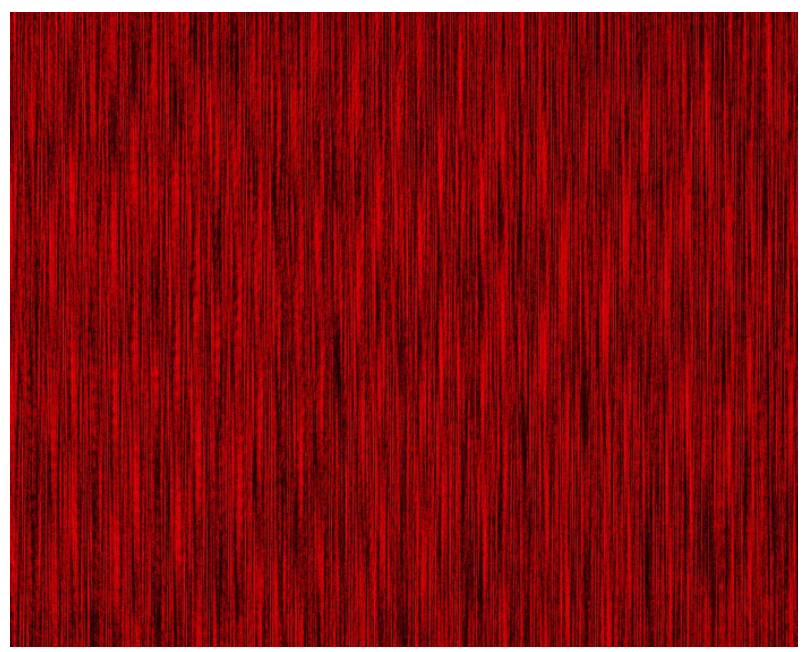

(e) 


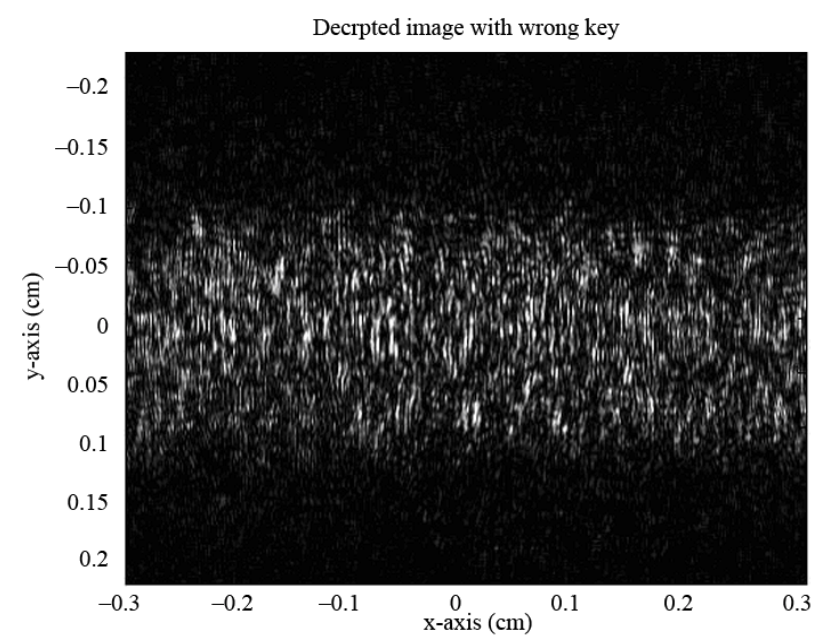

(f)

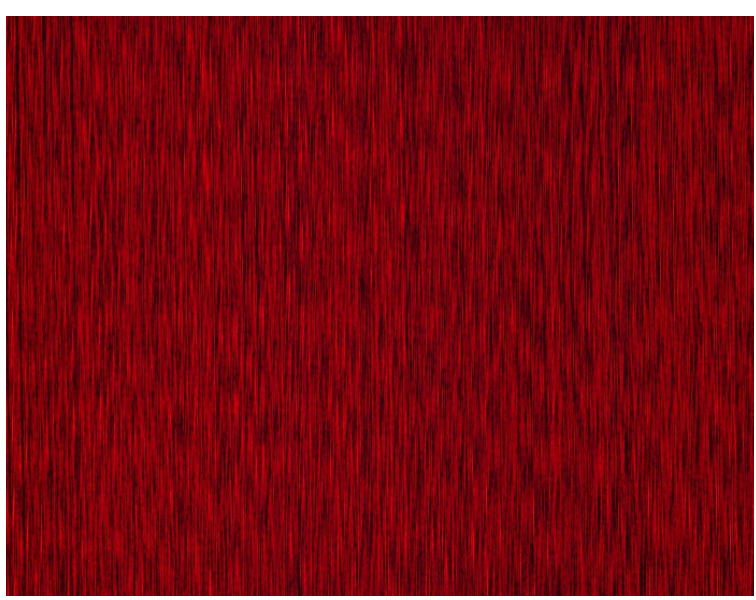

(g)

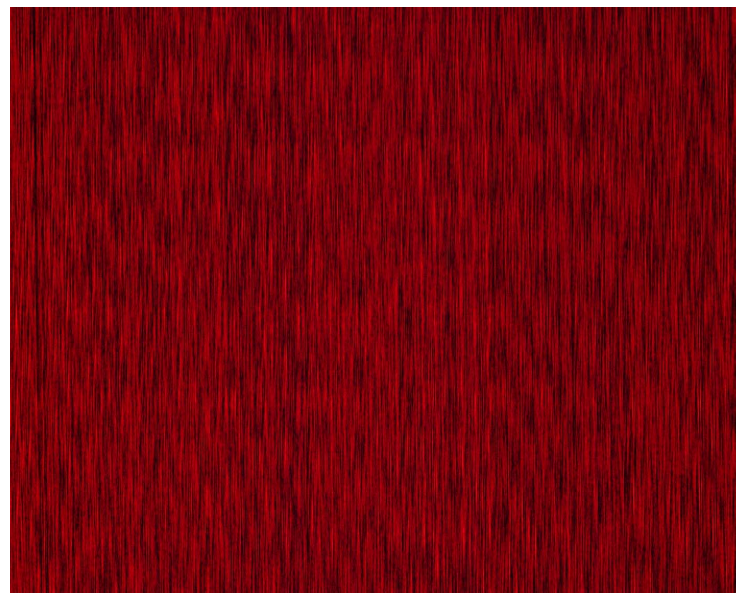

(h)

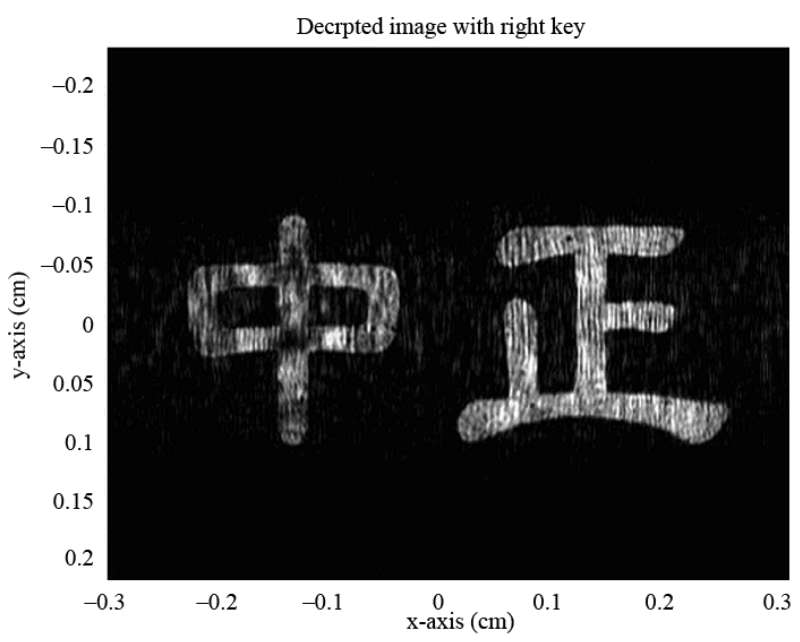

(i)

Figure 7. Experimental results: (a) first encrypted hologram without phase-step; (b) second encrypted hologram with phase-step; (c) decrypted image without key; (d) first wrong key hologram without phase-step; (e) second wrong key hologram with phase-step; (f) decrypted image with wrong key; (g) first right key hologram without phase-step; (h) second right key hologram with phase-step; (i) decrypted image with right key. 
mented using arbitrary two-step PSI with an unknown phase-step. The encrypted information that was encoded using some LPI number LLA was theoretically determined to be decryptable only using the correct LPI number or the right key and cannot be recovered without a key or with an incorrect LPI number. The number of degrees of freedom in the encryption scheme increase with the distances between the object and LLA from the $\mathrm{CCD}$, and also an unknown phase-step and the LPI number of the LLA. Therefore, the confidential information is not easily accessible by an unauthorized person.

\section{Acknowledgements}

The authors would like to thank the National Science Council of the Republic of China, Taiwan, for financially supporting this research under Contract No. NSC 100-2221-E-451-007.

\section{References}

[1] J. W. Goodman and R. W. Lawrence, "Digital Image Formation from Electronically Detected Hologram," Applied Physics Letters, Vol. 11, No. 3, 1967, pp. 77-79. doi:10.1063/1.1755043

[2] U. Schnars and W. Juptner, "Direct Recording of Holograms by a CCD Target and Numerical Reconstruction," Applied Optics, Vol. 33, No. 2, 1994, pp. 179-181. doi:10.1364/AO.33.000179

[3] I. Yamaguchi and T. Zhang, "Phase-Shifting Digital Holography," Optics Letters, Vol. 22, No. 16, 1997, pp. 1268-1270. doi:10.1364/OL.22.001268

[4] B. Javidi, G. Zhang and J. Li, "Experimental Demonstration of the Random Phase Encoding Technique for Image Encryption and Security Verification," Optical Engineering, Vol. 35, No. 9, 1996, pp. 2506-2512. doi: $10.1117 / 1.600854$

[5] B. Javidi and T. Nomura, "Securing Information by Use of Digital Holography," Optics Letters, Vol. 25, No. 1, 2000, pp. 28-30. doi:10.1364/OL.25.000028

[6] E. Tajahuerce, O. Matoba, S. C. Verrall and B. Javidi, "Optoelectronic Information Encryption with Phase-Shifting Interferometry," Applied Optics, Vol. 39, No. 14, 2000, pp. 2313-2320. doi:10.1364/AO.39.002313

[7] E. Tajahuerce and B. Javidi, "Encrypting Three-Dimensional Information with Digital Holography," Applied Optics, Vol. 39, No. 35, 2000, pp. 6595-6601. doi:10.1364/AO.39.006595

[8] S. Lai and M. A. Neifeld, "Digital Wavefront Reconstruction and Its Application to Image Encryption," $O p$ tics Communications, Vol. 178, No. 4-6, 2000, pp. 283289. doi:10.1016/S0030-4018(00)00641-6

[9] E. Tajahuerce, J. Lancis, B. Javidi and P. Andres, "Optical Security and Encryption with Totally Incoherent Light," Optics Letters, Vol. 26, No. 10, 2001, pp. 678-
680. doi:10.1364/OL.26.000678

[10] B. M. Hennelly, J. T. Sheridan, "Image Encryption and The Fractional Fourier Transform," Optik, Vol. 114, No. 6, 2003, pp. 251-265.doi:10.1078/0030-4026-00257

[11] R. Arizaga., R. Henao, R. Torroba, "Fully Digital Encryption Technique," Optics Communications, Vol. 221, No. 1-3, 2003, pp. 43-47. doi:10.1016/S0030-4018(03)01462-7

[12] L. Z. Cai, M. Z. He, Q. Liu, and X. L. Yang, "Digital Image Encryption and Watermarking by Phase-Shifting Interferometry," Applied Optics, Vol. 43, No. 15, 2004, pp. 3078-3084. doi:10.1364/AO.43.003078

[13] N. K. Nishchal, J. Joseph and K. Singh, "Fully Phase Encryption Using Digital Holography," Optical Engineering, Vol. 43 No. 12, 2004, pp. 2959-2966. doi: $10.1117 / 1.1811085$

[14] T. J. Naughton and B. Javidi, "Compression of Encrypted Three-Dimensional Objects Using Digital Holography," Optical Engineering, Vol. 43, No. 10, 2004, pp. 22332238. doi:10.1117/1.1783280

[15] A. Carnicer, M. M.-Usategui, S. Arcos, and I. Juvells, "Vulnerability to Chosen-Cyphertext Attacks of Optical Encryption Schemes Based on Double Random Phase Keys," Optics Letters, Vol. 30, No. 13, 2005, pp. 1644 1646. doi:10.1364/OL.30.001644

[16] L. Chen and D. Zhao, "Optical Image Encryption with Hartley Transforms," Optics Letters, Vol. 31, No. 23, 2006, pp. 3438-3440. doi:10.1364/OL.31.003438

[17] X. F. Meng, L. Z. Cai, X. F. Xu, X. L. Yang, X. X. Shen, G. Y. Dong and Y. R. Wang, "Two-Step Phase-Shifting Interferometry and Its Application in Image Encryption," Optics Letters, Vol. 31, No. 10, 2006, pp. 1414-1416. doi:10.1364/OL.31.001414

[18] X. Peng, H. H. Wei and P. Zhang, "Chosen-Plaintext Attack on Lensless Double-Random Phase Encoding in the Fresnel Domain," Optics Letters, Vol. 31, No. 22, 2006, pp. 3261-3263. doi:10.1364/OL.31.003261

[19] X. C. Cheng, L. Z. Cai, Y. R. Wang, X. F. Meng, H. Zhang, X. F. Xu, X. X. Shen, and G. Y. Dong, "Security Enhancement of Double-Random Phase Encryption by Amplitude Modulation," Optics Letters, Vol. 33, No. 14, 2008, pp. 1575-1577. doi:10.1364/OL.33.001575

[20] X. F. Meng, L. H. Cai, X. F. Xu, X. L. Yang, X. X. Shen, G. Y. Dong and H. Zhang, "Full-Phase Image Encryption by Two-Step Phase-Shifting Interferometry," Optik, Vol. 119, No. 9, 2008, pp. 434-440. doi:10.1016/j.ijleo.2007.02.003

[21] A. Nelleri, J. Joseph and K. Singh, "Lensless Complex Data Encoding for Digital Holographic Whole Information Security," Optical Engineering, Vol. 47, No. 11, 2008, pp. 115801-115809. doi:10.1117/1.3028351

[22] G. L. Chen, W. K. Yang, J. C. Wang and C. C. Chang, "Deterministic Phase Encoding Encryption in Single Shot Digital Holography," Applied Physics B, Vol. 93, No. 2-3, 2008, pp. 473-479. doi:10.1007/s00340-008-3201-5

[23] Y. Zhang and B. Wang, "Optical Image Encryption 
Based on Interference," Optics Letters, Vol. 33, No. 21, 2008, pp. 2443-2445. doi:10.1364/OL.33.002443

[24] Y. L. Xiao, X. Zhou, Q. H. Wang, S. Yuan, and Y. Y. Chen, "Optical Image Encryption Topology," Optics Letters, Vol. 34, No. 20, 2009, pp. 3223-3225. doi:10.1364/OL.34.003223

[25] O. Matoba, E. Tajahuerce, E. Perez-Cabre, M. S. Millan and B. Javidi, "Optical Techniques for Information Security," Proceedings of the IEEE, Vol. 97, No. 6, 2009, pp. 1128-1148. doi:10.1109/JPROC.2009.2018367

[26] W. T. Hsieh, M. K. Kuo, H. F. Yau and C. C. Chang, "A Simple Arbitrary Phase-Step Digital Holographic Reconstruction Approach without Blurring Using Two Holograms," Optical Review, Vol. 16, No. 4, 2009, pp. 466471. doi:10.1007/s10043-009-0090-8

[27] R. B. Johnson and G. A. Jacobsen, "Advances in Lenticular Lens Arrays for Visual Display," Proceedings of SPIE, Current Developments in Lens Design and Optical Engineering VI, San Diego, 2-4 August 2005, paper No. 5874-06.

[28] C. C. Chang, G. L. Chen, W. K. Yang, C. Y. Lin, H. F. Yau, "Deterministic Phase Encoded Holographic Data
Storage Using Lenticular Lens Array," Optical Review, Vol. 14, No. 4, 2007, pp. 214-218. doi:10.1007/s10043-007-0214-y

[29] T. C. Poon and P. P. Banerjee, "Contemporary Optical Image Processing with MATLAB," Elsevier, New York, 2001, pp. 4-6.

[30] "Pacur Lenstar Lenticular," 2005. http://www.pacur.com/pdf/lenstar.pdf

[31] J. W. Goodman, "Introduction to Fourier Optics," 2nd Edition, McGraw-Hill, Singapore, 1996, pp. 66-67.

[32] Y. Takaki, H. Kawai, and H. Ohzu, "Hybrid Holographic Microscopy Free of Conjugate and Zero-Order Images," Applied Optics, Vol. 23, No. 15, 1999, pp. 4990-4996. doi:10.1364/AO.38.004990

[33] U. Schnars and W. Juptner, "Digital Recording and Numerical Reconstruction of Holograms," Measurement Science and Technology, Vol. 13, No. 9, 2002, pp. R85R101. doi:10.1088/0957-0233/13/9/201

[34] W. K. Pratt, "Digital Image Processing," 4th Edition, Wiley, New York, 2007, pp. 759-760. doi:10.1002/9780470097434.app3 